\title{
Analysis of Touch Interaction for the Control of Smart Phones
}

\author{
Youngjae Lim ${ }^{1}$, Eui S. Jung ${ }^{1}$, Sungjoon Park ${ }^{2}$, Jaekyu Park ${ }^{1}$ \\ ${ }^{1}$ School of Industrial Management Engineering, Korea University, Seoul, Korea \\ ${ }^{2}$ Department of Industrial and Management Engineering, Namseoul University, Cheonan, Korea \\ ergoim@korea.ac.kr
}

\begin{abstract}
This study mainly aims to investigate characteristics of touch input. In addition, the touchable area that yields a specific hit rate is suggested for effective touch control. To achieve these goals, an empirical test was conducted through smart phone interface. The layout $(3 \times 4,4 \times 5,5 \times 6$ and $6 \times 8)$ and icon ratio $(0.5,0.7$ and 0.9$)$ were manipulated as an independent variable. A number of measurements (input offset, task completion time and preference) were utilized to assess the controllability of icon design. As a result, the layout and icon ratio were statistically significant on all three measurements. The $3 \times 4$ and $4 \times 5$ layouts were shown to have better performance. The icon ratio of 0.7 and 0.9 was also shown to have better preference. Meanwhile, the touchable area was derived based on the distribution of touch input. The general shape of the touchable area was vertically long rectangle. Based on the results of this study, the locations of the central region indicated a pattern of vertical touch and showed better performance. It was also shown that task completion time increased with an increase in the icon ratio while user preference became higher. This research is expected to contribute to practical suggestions for the interaction with an index finger.
\end{abstract}

Keywords: Finger input, Touch icon, Touch interaction, Touchable area, Controllability

\section{Introduction}

As product functions become more varied and complicated, users require a natural and convenient user interface. The natural user interface (NUI) takes into account the user's experience which shows how to efficiently create interfaces that reflect human capabilities [1]. The touch interface is modeled based on the behavior of users in real-world situations and is therefore regarded as one of the NUI.

The touch user interface (TUI) has several advantages in regards to input technology and user interaction [2]. For instance, the TUI provides new functions or applications such as the touch sensor screen, E-mail and internet browsing. Ergonomic features in regards to using mobile touch devices are related to finger input property, user tasks and input method like the users of thumb or index finger [3]. Furthermore, the controllability of touch icon has to be properly considered from the perspective of customeroriented design.

With the trend of wide and various mobile applications, smart phones appear to be the typical mobile touch device. One major feature of smart phones has been the adoption of the touch screen. This is due to the touch interface being intuitive, providing software flexibility and space saving [4]. In addition, the global market for smart phones is expected to expand to 700 million units by 2015. The utilization of the smart phone enables more flexible and capable touch interaction compared to typical feature phone which is limited to the physical button. Therefore, designers should make in-depth studies of the touch behavior and design considerations for touch icon.

After reviewing the issues related to the touch interaction and icon design, it is shown that a variety of the studies have investigated touch-based usability. Since the 1980s, researchers have been concerned with suitable touch key design under large screen environment such as ATMs or PDAs. The main aspects of these studies were the effect of the touch key size [5] and the space between touch keys [6]. More recently, there has been relevant research focusing on interaction techniques regarding the accuracy of touch [7] and performance of touch screen keyboards using a smart phone [8]. However, there were very few design guidelines regarding the user characteristics for finger interactions and icon design with mobile touch devices especially in smart phones.

There are apparent needs for an investigation into the icon design and touch interaction in order to enhance touch controllability. This study mainly aims to investigate the characteristics of touch input according to the interface design. Furthermore, the touchable area that yields a desirable hit rate is suggested for effective touch control. The results of this study will contribute to propose practical suggestions for interactions with an index finger.

\section{Design approach for TUI}




\subsection{Finger input property}

To improve the controllability of smart phone, the finger input property needs to be explained. The finger input property and ability influence on the accuracy of touch. Figure 1 illustrates the characteristics of finger input.

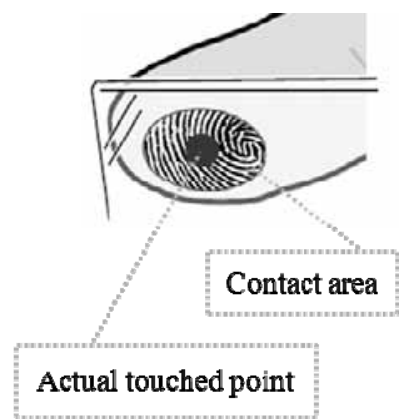

Figure 1. Finger input property on touch screen

The contact area indicates an ellipsoidal zone which covers entire area between finger and device. Current smart phones are mostly adopted on capacitive touch screen. It is assumed that users select targets with the centroid of the contact area. This centroid of the contact area is defined as an actual touch point. Moreover, the input offset means a gap between desired target and actual touched point.

\subsection{Design Considerations}

The size of targets for visual tasks can be traditionally understood from two aspects; objective size and subjective size [9]. The objective size is defined by measuring the geometric dimensions of the icon by width times length. On the other hand, the subjective size is defined by an ellipsoidal area which indicates a set of actual touch points as seen in Figure 2.

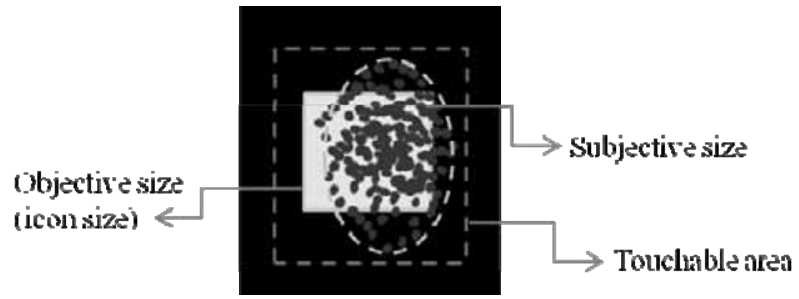

Figure 2. Design dimensions regarding touch icon

The understanding of difference between these two aspects has become more important as the usage of smart phone has increased. Furthermore, the touchable area was derived as a rectangular form for design purpose based on a distribution of the touch input.

\section{Method}

\subsection{Subjects}

A total of forty people voluntarily participated in the experiment. The age of the participants varied between 22 and 36 years with a mean of 27.5 years. They had an average of 17.7 months of experience with mobile touch screen device and 8.8 months with smart phones.

\subsection{Experimental design}

The layout which means the number of icons in a matrix form was selected as one of the independent variables. A total of four different layouts $(3 \times 4,4 \times 5$, $5 \times 6$ and $6 \times 8$ ) were evaluated on the ease of control. The icon ratio was another independent variable that varied at three levels; $0.5,0.7$ and 0.9 . The icon ratio was defined as a ratio of the width of icon $\left(\mathrm{W}_{\mathrm{I}}\right)$ to the width of layout size $\left(\mathrm{W}_{\mathrm{L}}\right)$ as shown in Figure 3.

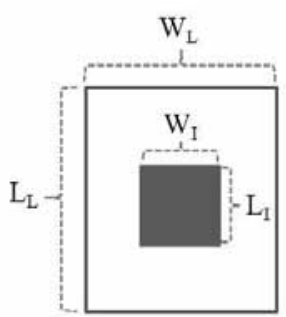

Icon size $\left(\mathrm{W}_{\mathrm{I}} \times \mathrm{L}_{\mathrm{I}} ; \mathrm{W}_{\mathrm{I}}=\mathrm{L}_{\mathrm{I}}\right)$

Icon ratio $\left(\mathrm{W}_{\mathrm{I}} / \mathrm{W}_{\mathrm{L}}\right)$

Layout size $\left(\mathrm{W}_{\mathrm{L}} \times \mathrm{L}_{\mathrm{L}} ; \mathrm{W}_{\mathrm{L}}=\mathrm{W}_{\mathrm{D}} /\right.$ \# of icons placed horizontally)

Figure 3. Definitions of layout and icon ratio

The dependent variables were classified into two types; touch performance and subjective assessment. The touch performance included two measures on input offset and task completion time. Furthermore, the subjective measure was assessed as a preference score.

\subsection{Tasks}

The icons used in this experiment were a square within which a functional image was shown. The priority for the functions was determined based on the frequent usage of smart phone users. From the survey of frequent usage, the icons such as Appstore, Books, Mobile and SMS were then selected.

The tasks in the main experiment were to find a target icon and execute a right function on the home screen of the smart phone. A series of tasks were conducted through the combination of finding a right function, shape and color, respectively.

\subsection{Environment and Procedure}

The experiment was conducted in a quiet laboratory. Each participant was required to perform the task alone with no other participants. The smart phone used in the study is HTC Desire HD with a display size of 4.3 inch touch screen. The participants performed the tasks as naturally as they could, favoring accuracy to speed. In this experiment, the participants held a smart phone with one-hand and used the index finger of the other hand for touch input as described in Figure 4. All the trials were fully randomized within each experimental condition using the Latin-square balancing technique. 


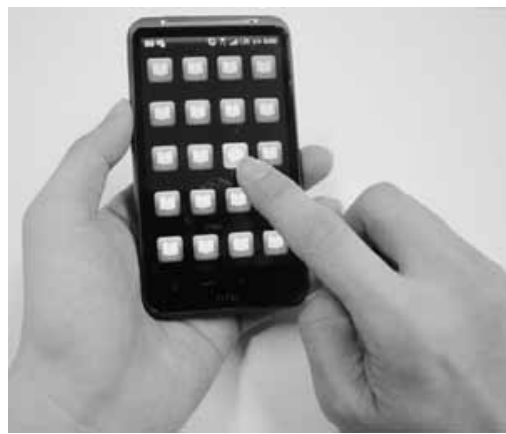

Figure 4. Experimental apparatus

\section{Results}

\subsection{Input offset}

A total of 138,240 (12 experimental conditions x 3 series of tasks x 96 trials $\times 40$ subjects) input data were gathered from the experiment. Table 1 indicates that most of the mean values in $\mathrm{X}$ and $\mathrm{Y}$ offsets were positive and negative, respectively. That is the subjects tend to input in a direction to the right and downward from the center. The standard deviation (SD) of X and $\mathrm{Y}$ offsets varied according to the each experimental condition as seen in Table 1. Especially, the SD variation of $\mathrm{Y}$ offset was relatively greater than that of $\mathrm{X}$ offset. This characteristic revealed that the range of input offset in $\mathrm{Y}$ axis was greater than that in $\mathrm{X}$ axis. Therefore, the touchable area in this study is suggested as a vertically long rectangle.

Table 1. Descriptive statistics of input offset

\begin{tabular}{c|c|c|c|c|c}
\hline \multicolumn{2}{c|}{ Experimental factor } & \multicolumn{2}{c|}{$\mathrm{X}$ offset $(\mathrm{mm})$} & \multicolumn{2}{c}{ Y offset $(\mathrm{mm})$} \\
\hline Layout & Icon Ratio & mean & $\mathrm{SD}$ & mean & $\mathrm{SD}$ \\
\hline \multirow{3}{*}{$3 \times 4$} & 0.5 & 0.54 & 2.05 & -0.28 & 3.34 \\
\cline { 2 - 6 } & 0.7 & 0.63 & 1.85 & -0.69 & 2.33 \\
\cline { 2 - 6 } & 0.9 & 0.31 & 2.31 & -0.63 & 2.52 \\
\hline \multirow{3}{*}{$4 \times 5$} & 0.5 & 0.00 & 2.26 & -1.09 & 3.86 \\
\cline { 2 - 6 } & 0.7 & 0.04 & 2.59 & -0.85 & 2.69 \\
\cline { 2 - 6 } & 0.9 & -0.07 & 2.20 & -0.74 & 2.25 \\
\hline \multirow{3}{*}{$5 \times 6$} & 0.5 & 0.32 & 1.76 & -1.15 & 3.26 \\
\cline { 2 - 6 } & 0.7 & 0.43 & 2.01 & -1.17 & 2.61 \\
\cline { 2 - 6 } & 0.9 & 0.40 & 2.05 & -0.88 & 2.07 \\
\hline \multirow{3}{*}{$6 \times 8$} & 0.5 & 0.56 & 1.99 & -0.49 & 3.36 \\
\cline { 2 - 6 } & 0.7 & 0.45 & 1.68 & -0.55 & 2.07 \\
\cline { 2 - 6 } & 0.9 & 0.59 & 1.93 & -0.34 & 2.01 \\
\hline
\end{tabular}

\subsection{Task completion time}

Task completion time was analyzed using the $4 \times 3$ ANOVA. The main effect of layout $(\mathrm{F}$-value $=121.31, \mathrm{p}$ $<0.0001)$ and icon ratio (F-value $=315.24, \mathrm{p}<0.0001)$ were observed to be significant. An interaction effect between layout and icon ratio did not appear. The posthoc analysis was conducted on the significant main effects.

Figure 5 indicates that the $5 \times 6$ and $6 \times 8$ layouts significantly differed from other conditions. The $6 \times 8$ layout showed a worst performance. Meanwhile, a difference of the task completion time among all levels of icon ratio was statistically significant. The subjects took more time completing the task as the icon ratio increased in each layout.

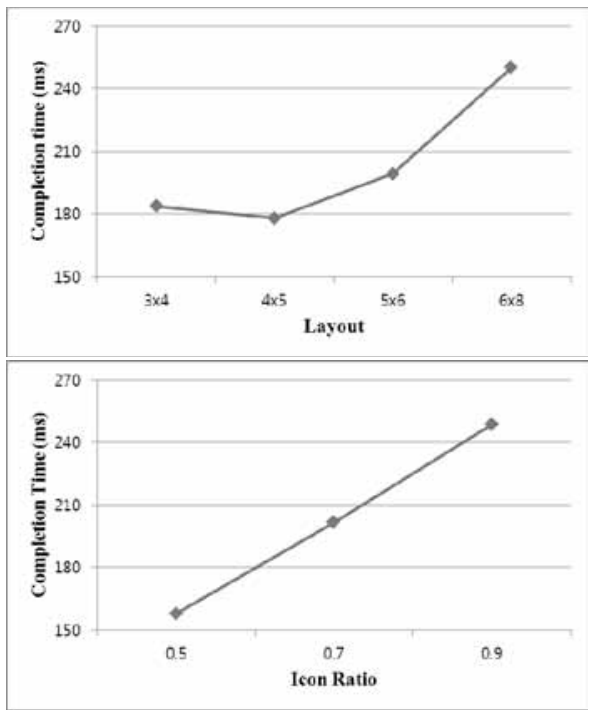

Figure 5. Mean completion time of each condition

\subsection{Preference}

The $4 \times 3$ ANOVA and SNK tests were also conducted to analyze this assessment. The main effect of layout $(\mathrm{F}$ value $=131.658, \mathrm{p}<0.0001)$ and icon ratio $(\mathrm{F}$-value $=$ $36.389, \mathrm{p}<0.0001)$ were statistically significant. From the results of SNK tests, the all levels of layout had significant differences.

Figure 6 describes that the preference for the $6 \times 8$ layout had the lowest score in terms of the ease of control. Additionally, the icon ratios between 0.7 and 0.9 in all layout conditions did not show any significant differences. The preference for the icon ratio of 0.5 was significantly lower than that for the icon ratios of 0.7 and 0.9 .

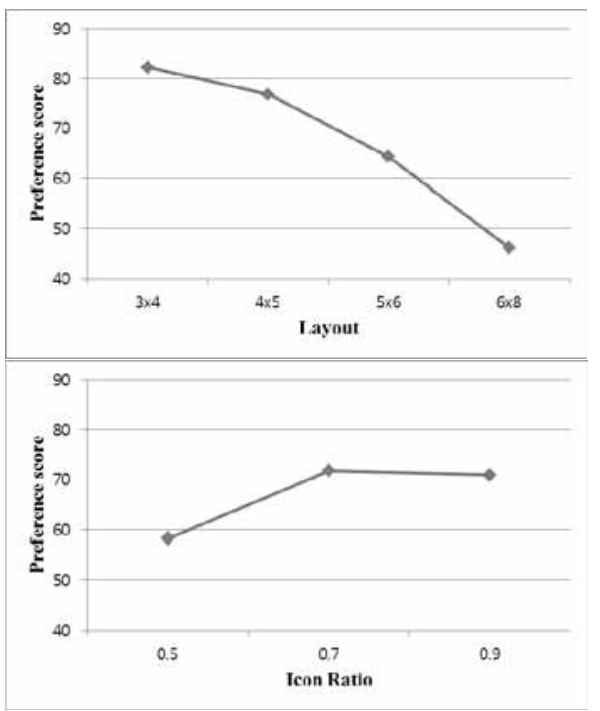

Figure 6. Mean preference score of each condition 


\subsection{Suggestion for the touchable area}

As mentioned previously, the touchable area was regarded as a rectangular form in order to enhance the practical applicability in designing interfaces while the subjective size was an ellipse. Furthermore, the size of touchable area was determined according to the desirable hit rate which guaranteed a specific controllability.

In order to derive the touchable area, the bivariate normal distribution of input offset was considered. The shape of the distribution was determined in terms of individual parameters $\left(\mu_{\mathrm{x}}, \mu_{\mathrm{y}}, \sigma_{\mathrm{x}}\right.$ and $\left.\sigma_{\mathrm{y}}\right)$. Moreover, contours of iso-density for the bivariate normal distribution were also obtained as illustrated in Figure 7.

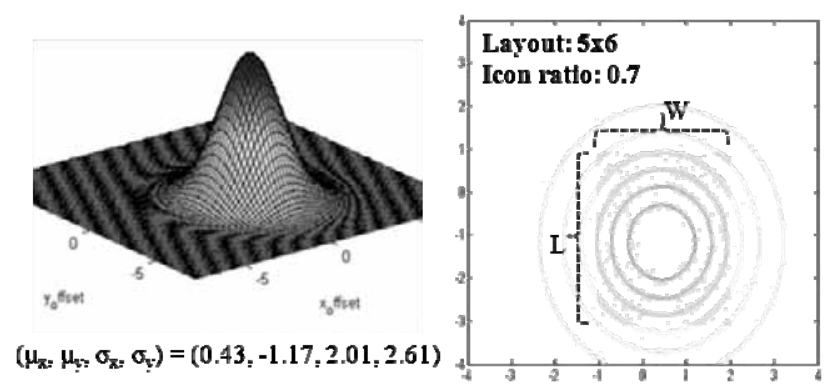

Figure 7. Distribution and constant density ellipse

Figures 7 and 8 depict an example of derivation under the condition of 5x6 layout and icon ratio of 0.7 . The ratio between width (W) and length (L) of the touchable area was maintained with the value of $\sigma_{\mathrm{y}} / \sigma_{\mathrm{x}}$. Since the constant density ellipse was established with the ratio of major axis to minor axis corresponding to $\sigma_{\mathrm{y}} / \sigma_{\mathrm{x}}$. Therefore, the ratio of $1.30(\mathrm{~L} / \mathrm{W})$ of the touchable area was calculated by $2.61 / 2.01$.

The desirable hit rate is a criterion of icon design to guarantee a goal of touch accuracy predetermined by the designer. When the desirable hit rate was set to be $90 \%$, the results of derivation for icon design were described as shown in Figure 8. The length of $10.44 \mathrm{~mm}$ of the touchable area was calculated by $8.03 \mathrm{~mm}$ (width of the touchable area) times $1.30\left(\sigma_{\mathrm{y}} / \sigma_{\mathrm{x}}\right)$.

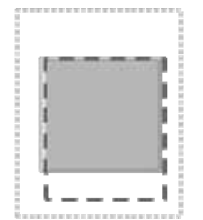

Layout size (11.52 mm X $15.48 \mathrm{~mm})$

Icon size ( $8.03 \mathrm{~mm}$ X $8.03 \mathrm{~mm})$

$\mathrm{LI}^{-}$Touchable area $(8.03 \mathrm{~mm}$ X $10.44 \mathrm{~mm})$

Figure 8. Derivation result of the touchable area

\section{Discussion and Conclusion}

This study conducted a test of touch interaction with an index finger on smart phone interface. The results of the test revealed that the average input offset was influenced by the major hand of users. Furthermore, the $4 \times 5$ layout and icon ratio of 0.7 were shown to be a best design, considering for both user performance and preference.
Meanwhile, we could discuss the characteristics of input offset and touch controllability regarding the adequacy of user interface. First, the lower region of the smart phone had smaller X offsets. This was due to users being more conscious of physical limitations which were known as the frame effect. In addition, the locations of the central region indicated a pattern of vertical touch. This explains that the index finger touched the icon vertically like a stylus pen while approaching to the central region.

Second, the distinctive result in terms of touch performance was identified. The task completion time increased with an increase in the icon ratio. In other words, the greater icon size did not always guarantee better performance. However, previous study indicated that greater key size usually resulted in a better performance [10]. The reason for difference between the results would be a space limitation for smart phone.

This research also suggested the touchable area for designing a touch icon which guaranteed predetermined touch accuracy. The guideline to the touchable area would apply to a design of grid layouts for smart phone.

\section{References}

[1] Benko, H., Wilson, A., and Baudisch, P.: Precise selection techniques for multi-touch screens, conf. Human Factors in Computing Systems, 1263-1272, 2006

[2] Burnett, D., Coulton, P., and Lewis, A.: Providing both physical and perceived affordances using physical games pieces on touch based tablets, proc. Interactive Entertainment: Playing the System, 2012

[3] $\mathrm{Au}$ O. K.-C., Tai C.-L.: Multitouch finger registration and its applications, proc. Computer-Human Interaction, 41-48, 2010

[4] Colle, H.A., and Hiszem, K.J.: Standing at a kiosk: effects of key size and spacing on touch screen numeric keypad performance and user preference, Ergonomics, 47: 1406-1423, 2004

[5] Scott, B., and Conzola, V.: Designing Touch Screen Numeric Keypads: Effects of Finger Size, Key Size, and Key Spacing, proc. Human Factors and Ergonomics Society, 41(1): 306-364, 1997

[6] Martin, G.L.: Configuring a numeric keypad for a touch screen, Ergonomics, 31: 945-953

[7] Xu, W., Yu, C., and Shi, Y.: Regional Sliding: enhancing target selection on touch screen-based mobile devices, proc. CHI EA, 1261-1266, 2011

[8] Song, J., Ryu, T., Bhan, S., and Yun, M.H.: Performance analysis of text entry with preferred one hand using smartphone touch keyboard, proc. Human Factors and Ergonomics Society, 41(1): 306-364, 1997

[9] Watson, H.D.: The effects of objective and perceived size properties on visual form matching, Experimental Psychology, 110(4): 547-567, 1981

[10] Jin, Z., Plocher, T., and Kiff, L.: Touch screen user interfaces for older adults: Button size and spacing, Lecture Notes in Computer Science, 4554: 933-941, 2007 